\title{
Fermion Condensates in Two Colours Finite Density QCD at Strong Coupling
}

\author{
R. Aloisio ${ }^{a, d}$, V. Azcoiti ${ }^{b}$, G. Di Carlo ${ }^{c, d}$, A. Galante ${ }^{a, d}$ and A.F. Grillo ${ }^{d}$ \\ ${ }^{a}$ Dipartimento di Fisica dell'Università di L'Aquila, 67100 L'Aquila, (Italy). \\ ${ }^{b}$ Departamento de Física Teórica, Facultad de Ciencias, Universidad de Zaragoza, \\ 50009 Zaragoza (Spain). \\ ${ }^{c}$ Istituto Nazionale di Fisica Nucleare, Laboratori Nazionali di Frascati, \\ P.O.B. 13 - 00044 Frascati, (Italy). \\ ${ }^{d}$ Istituto Nazionale di Fisica Nucleare, Laboratori Nazionali del Gran Sasso, \\ 67010 Assergi (L'Aquila), (Italy).
}

\begin{abstract}
We study unquenched lattice $S U(2)$ at nonzero chemical potential at strong coupling and with eight flavours of Kogut-Susskind fermions. Introducing a diquark source term we analyze the behaviour of different types of fermion condensates. Using a non standard approach we can obtain results at zero external source without extrapolations. We find strong evidences for a (high density) second order phase transition where a diquark condensate appears. The corresponding critical chemical potential is in good agreement with half the pion mass.
\end{abstract}




\section{Introduction}

The behaviour of hadronic matter at high density is very interesting from many points of view: in astrophysics is relevant for the study of neutron stars and extremely compact objects, in cosmology is crucial for the understanding of phase transitions in the early Universe. The new states of matter should be partially accessible to direct experimental verification at the next generation of heavy ions collision experiments.

New ideas concerning the high density regime of QCD have been recently proposed [1]; in this regime new forms of ordering are expected to appear. Asymptotic freedom of QCD implies that high density baryonic matter has a behaviour similar to a nearly ideal Fermi gas of quarks; in this gas, due to the interaction, a (weak) attractive channel is expected between quarks of different colours that could lead to the formation of quark pairs (analogous of Cooper pairing in solid state systems at low temperature). The formation of quark pairs breaks the local $S U(3)$ colour symmetry of the theory, analogously to the breaking of electromagnetic gauge invariance in superconductivity, and therefore the name "colour superconductivity". The breaking of $S U(3)$ local symmetry may be regarded as a Higgs mechanism, so one may expect that some gluons become massive [1].

These features of finite density QCD can be studied only nonperturbatively. Unfortunately the lattice approach, the most powerful tool to perform first principles, nonperturbative studies, is affected in the case of finite density QCD by the well known sign problem that has prevented until now any step towards its understanding beyond the mean field results.

This problem does not affect a class of gauge theories at finite baryonic density, i.e. those with fermions in the pseudo-real representation [2]. In particular the study of two colours QCD (i.e. the $S U(2)$ gauge theory) is feasible also at finite density and may produce some interesting insights for the $S U(3)$ case. The use of $S U(2)$ as gauge group, in fact, offers the possibility of performing direct simulations of the finite density theory, since quarks and antiquarks belong to the same (real) representation of the group and the fermionic determinant is real and positive also for nonzero chemical potential. In two colours QCD the formation of quark-quark pairs does not break the colour symmetry of the theory $(S U(2)$ pairs are colourless), and may be regarded as a spontaneous breaking of baryon number conservation that does not imply a Higgs mechanism as in the $S U(3)$ case.

In this work we present a new simulation scheme for unquenched $S U(2)$ at finite density particularly efficient for studying the observable dependence 
on the diquark source term. To have the possibility to compare our simulations with existing ones we have to consider the $\beta=0$ limit since only in this case reliable results are available allowing a straightforward test of our numerical procedure. We consider 8 flavours of Kogut-Susskind fermions to avoid sign ambiguities (see below) in the determination of the fermionic partition function.

The study of diquark condensation is made possible by the introduction, in the fermionic matrix, of an explicit source term of the type:

$$
\sum_{x}\left(J q_{x} q_{x}+\bar{J} \bar{q}_{x} \bar{q}_{x}\right) .
$$

In the limit $m=0, \mu=0$ and $J=\bar{J}=0$ the diquark and chiral condensates are degenerate (i.e. there is a symmetry transformation which turns the chiral condensate in the diquark one) and the theory with staggered fermions has a global $U(2)$ symmetry [3]. Introducing a nonzero chemical potential the diquark and chiral condensates are no longer degenerate and the $U(2)$ symmetry is reduced to $U_{V}(1) \otimes U_{A}(1)$ : the vector symmetry is associated to the conservation of the baryon number and the axial one is the chiral symmetry. The presence of a nonzero diquark condensate in the $S U(2)$ theory indicates a spontaneous breaking of the $U_{V}(1)$ vector symmetry .

Two colours QCD in the strong coupling limit has been studied numerically using different algorithms for dynamical fermions: the Monomer Dimer (MD) algorithm [4, 5], the Monomer Dimer Polymer (MDP) algorithm [6] and, more recently, a more standard Hybrid Monte Carlo [7]. While MDP simulations are restricted to the $\beta=0$ case, the HMC algorithm has problems when the diquark source term has to be included in the generation of configurations so that $J \neq 0$ results are partially quenched.

The MD algorithm allows the simulation of $S U(N)$ theories for $N$ even. In this approach a source like (1), which allows the study of the transition to a phase in which the diquark condensate is nonzero, is necessary also to ensure the convergence of the algorithm. In [0], using a quark mass of 0.2 , the authors obtain a phase diagram in which, with increasing $\mu$, a phase with $<q q>\neq 0$ and $<\bar{q} q>\simeq 0$ appears. This phase may be identified with the expected phase of diquark formation.

A study of diquark condensation in (partially quenched) $S U(2)$ at finite density at $J \neq 0$ and finite coupling can be found in [7, 8]; working explicitly

\footnotetext{
${ }^{1}$ This is not in contrast with the Vafa-Witten theorem that prevents a spontaneous breaking of a vectorial symmetry because in this case the massless Dirac operator of the theory is not antihermitian.
} 
at $J \neq 0$ one has to face the problem of extrapolating the results to the $J=0$ limit; this extrapolation can be a source of systematic errors.

In this work we have analyzed the issue of diquark condensation from a point of view inherited from our previous study of chiral symmetry breaking in noncompact $Q E D$ [9]. This framework, based on the study of susceptibilities, allows a shorter path from numerical data to physical conclusions, Within our approach these observables can be calculated at zero source, then avoiding part of the (potentially) harmful extrapolation procedures. Where possible and meaningful, we will also try a direct comparison of numerical lattice results with the prediction of the effective low energy Lagrangian for generic QCD-like theories as reported in [2].

The paper is planned as follows: in section 2 we present our approach and the simulation scheme and in section 3 we discuss our numerical results on chiral as well as diquark observables.

\section{Simulation Scheme}

We have introduced in the action functional of staggered fermions a source term for the diquark. With this source term the fermionic sector of the partition function may be written formally as follows

$$
Z_{\text {ferm }}=\int d \psi d \bar{\psi} e^{\bar{\psi} M \psi+\psi J \psi+\bar{\psi} \bar{J} \bar{\psi}}
$$

where $M$ is the $2 V \times 2 V$ fermionic matrix at nonzero chemical potential and $J, \bar{J}$ are diquark and antidiquark source terms respectively ( $V$ is the lattice volume) .

In order to perform the integration over the fermion fields in eq. (2) it is necessary to rewrite the fermionic term in a bilinear form. This is possible introducing a two component Grassmann field of the form [\&]

$$
\phi=\left(\begin{array}{l}
\bar{\psi} \\
\psi
\end{array}\right) .
$$

The fermionic partition function then becomes

$$
Z_{\text {ferm }}=\int d \phi e^{\phi^{T} A \phi}=\operatorname{Pf}(A)
$$

where $\operatorname{Pf}(A)$ indicates the Pfaffian of the $4 V \times 4 V$ matrix $A$ 


$$
A=\left(\begin{array}{cc}
\bar{J} & \frac{M}{2} \\
-\frac{M^{T}}{2} & J
\end{array}\right) .
$$

We require the matrix $A$ to be antisymmetric and therefore we chose $J=\bar{J}=j \tau_{2}$ with $j$ a real number and $\tau_{2}$ a $V \times V$ block diagonal matrix with the second Pauli matrix on the diagonal. In the case of an antisymmetric matrix we may express the Pfaffian in terms of the determinant: $\operatorname{Pf}(A)=$ $\pm(\operatorname{Det}(A))^{1 / 2}$. This partition function describes $n_{f}=4$ quark flavours. In order to avoid ambiguities in the definition of the sign we consider a theory with $n_{f}=8$. As suggested by the low energy effective theory results, we do not expect this choice to affect significantly the physics. This indeed is what happens in the continuum limit where the dependence on $n_{f}$ is very mild [2]

In this case the fermionic partition function becomes $Z_{\text {ferm }}=\operatorname{Det}(A)$ that may be expressed as a polynomial in $j$ considering that

$$
\begin{aligned}
\operatorname{Det} A & =\operatorname{Det}\left(\begin{array}{cc}
j \tau_{2} & \frac{M}{2} \\
-\frac{M^{T}}{2} & j \tau_{2}
\end{array}\right)=\operatorname{Det}\left[\left(\begin{array}{cc}
0 & \frac{M \tau_{2}}{2} \\
-\frac{M^{T} \tau_{2}}{2} & 0
\end{array}\right)+j\left(\begin{array}{cc}
I & 0 \\
0 & I
\end{array}\right)\right] \\
& =\operatorname{Det}(\tilde{A}+j I)
\end{aligned}
$$

As we have pointed out in the introduction we have studied the phase structure of the theory in the limit of infinite gauge coupling $(\beta=0)$. Our idea is to use a Microcanonical Fermion Averaged inspired algorithm where the observables are calculated at fixed plaquette energy [12]. Since we are interested in the $\beta=0$ limit of the theory the numerical scheme simplifies because we can safely calculate our observables using random gauge configurations i.e. with only the Haar measure of the gauge group as a weight. This is possible because, according to the results reported in [7], that ensemble (which has a gaussian distribution of the plaquette energy around zero) has a net overlap with the importance sample of fixed energy gauge configurations corresponding to the values of $\mu$ and $m$ used in our calculations. To test this procedure we have recently compared directly the chiral condensate and the number density as obtained from HMC and MFA simulations $(j=0$ and $\beta=0$ ) finding a complete agreement between them [14.

We have computed $\operatorname{Det}(\tilde{A}+j I)$, with a library routine, diagonalising the matrix:

$$
\tilde{A}=\left(\begin{array}{cc}
0 & \frac{M \tau_{2}}{2} \\
-\frac{M^{T} \tau_{2}}{2} & 0
\end{array}\right)
$$


for a given set of $\mu$ and $m$. At this point, having determined the partition function for discrete $\mu$ and $m$ values and continuously in $j$, we can evaluate, using finite differences or explicit differentiation, the derivatives of the free energy in the thermodynamic parameters.

Diagonalising also the Dirac matrix $M$ (at $m=0$, the same $\mu$ values and the same gauge field configurations) we can study the dependence of the partition function continuously in the quark mass, hence complementing our analysis with chiral observables at $j=0$.

Our aim is to find the phase structure of the model in the $\mu-m$ plane and, possibly, characterize the various phases in term of breaking/restoration of the two global symmetries (vector/axial). The standard way to proceed in such an analysis is to compute the condensates introducing a symmetry breaking term (SBT), i.e. a mass or a $j$ term, and then extrapolate the results at zero value of the SBT. In finite volume calculations, the actual value at vanishing SBT is exactly zero ( e.g. the chiral condensate is an odd function of the mass ). Therefore there are severe limitations to the standard procedure arising from (in principle) unknown effects of the arbitrariness in the extrapolation scheme. To have a good control on these effects we should repeat the analysis for several different lattice volumes in order to disentangle true finite size effects from those arising from the extrapolation.

To avoid these problems we proceed in a different way: following a scheme developed successfully for the study of the abelian non-compact model [9], we looked at the second derivatives of the free energy with respect to the symmetry breaking source i.e. the susceptibilities. The main advantage is that in the symmetric phase the susceptibilities can be evaluated directly in the vanishing SBT limit, hence avoiding any extrapolation. A complementary and independent analysis based on the behaviour of the probability distribution functions of the order parameters [10] will be presented elsewhere [11].

We present, in the following, results for the diquark susceptibility and the chiral susceptibility at $j=0, m \neq 0$ as a function of the chemical potential $\mu$. Some comments are in order to explain why we used $m \neq 0$. What is expected from simplified phenomenological models is an onset transition at half the mass of the lightest baryon (corresponding to the transition at one third of the nucleon mass in the $\mathrm{SU}(3)$ case). This transition could be followed, at larger $\mu$ by a superconducting phase signalled by a non zero diquark condensate and (possibly) vanishing chiral condensate. The analysis of the chiral properties of this phase transition, in principle feasible even in the chiral limit owing to the use of the susceptibilities, is in practice impossible because the lightest baryon of the theory is a Goldstone boson, 
massless in the chiral limit. Then the critical chemical potential is expected to be zero.

We have instead chosen to work at non zero, but small, quark mass value, a situation that resembles more closely the physically interesting $S U(3)$ case: therefore the critical chemical potential is different from zero and the analysis of the transition is possible.

A drawback is that we loose the correspondence between deconfined and chiral symmetric phase, owing to the explicit SBT in the chiral sector (the quark mass). Using small values for the quark mass we can, however, expect a behaviour similar to the zero mass case, i.e. an essentially vanishing chiral condensate, with a non zero critical chemical potential. This will translate in a peak of the chiral susceptibility, signalling the developing of a symmetry restoration transition in the zero quark mass limit.

We can write the diquark susceptibility (at $j=0$ ) as

$$
\chi_{q q}(j=0)=\left\langle\frac{1}{V} \sum_{n=1}^{2 V} \frac{1}{\lambda_{n}^{2}}\right\rangle
$$

where $\lambda_{n}$ are the eigenvalues of $i \tilde{A}$, while for the chiral susceptibility we can proceed from the eigenvalues of the Dirac matrix $M$ to compute the free energy and then the second derivative in the quark mass $m$.

We want to stress here that relation (3) is exact only in the symmetric phase, where one can exchange the $j \rightarrow 0$ and the thermodynamical limit, in analogy with the discussion in [9] for the chiral susceptibility. Notwithstanding that the right-hand side of (3) has in any case a very clear physical meaning in both broken and unbroken phases. In fact as follows from an analysis of the probability distribution function of the order parameter similar to the one developed in [10], the right-hand side of (3) normalized by the lattice volume is, in the infinite volume limit, just the square of the vacuum expectation value of the diquark condensate (see equation (12) in [10]). Therefore the expectation value in equation (3), even if it does not give the susceptibility in the broken phase, diverges as the lattice volume if and only if we have diquark condensation, and this is an important result which can be used in the numerical simulations to identify the diquark condensation phase.

We have studied the theory in a $4^{4}$ and $6^{4}$ lattice diagonalising 300 gauge configurations in the first lattice volume and 100 in the second one. These simulations have been performed at quark mass $m=0.025,0.05,0.20$ and for values of the chemical potential ranging from $\mu=0$ to $\mu=1.0$. 
All numerical simulations have been performed on a cluster of Pentium II and PentiumPro at the INFN Gran Sasso National Laboratory.

\section{$3 \quad$ Numerical Results}

In this section we discuss our numerical results for strongly coupled $S U(2)$ gauge theory at finite chemical potential with $n_{f}=8$ quark flavours. Our numerical approach has been tested by checking that it produces results in agreement with those of the Monomer-Dimer algorithm reported in [4, [5].

We present first the results for chiral observables; as said before, working at a small but nonzero quark mass, we do not expect a true phase transition signal. A singularity in the chiral susceptibility will be present only in the chiral limit, while we are forced to work at non zero quark mass by the requirement of having a massive baryon (as in the $\mathrm{SU}(3)$ model). In any case we expect to see a signal in the susceptibility (see [9] for a similar analysis in a slightly different case).

In figure 1 we report the chiral susceptibility as a function of the chemical potential computed at $m=0.025$ and $m=0.05$ in the two lattice volumes $4^{4}$ and $6^{4}$. From this figure we see a crossover at a value of the chemical potential which depends on the quark mass: the chiral susceptibility presents a sharp peak, although his height does not increase significantly with the volume, as expected lacking a divergent correlation length.

The values of the (pseudo)critical chemical potential $\mu_{c}$ defined as the position of the maximum of the peak, are reported in table I together with the corresponding values of half the pion mass at $\mu=0$ computed using the approximated formula reported in [13].

\begin{tabular}{|c|c|c|}
\hline$m$ & $\mu_{c}$ & $\frac{m_{\pi}}{2}$ \\
\hline 0.025 & $0.20(3)$ & 0.19 \\
0.05 & $0.30(3)$ & 0.27 \\
\hline
\end{tabular}

Table I

We conclude that $\mu_{c}$ moves, varying the quark mass, in the expected way. We have also checked that at $m=0$ the critical chemical potential moves to zero.

In order to have a clearer readibility and an easier interpretation of the result we will analyze the inverse right-hand side of equation (3). Coming from the symmetric phase, this quantity must approache zero at the critical 
point. In a finite volume a nonzero value will in any case be found, but with increasing volumes it has to approach zero.

In figure 2 we report the inverse right-hand side of equation (3) at $j=0$ as a function of $\mu$ at $m=0.05$ in the two lattice volumes (see also Figure 3 for the results at a larger mass). For the larger volume, we can easily recognise three different regions in $\mu$. Starting from the value at zero chemical potential, the reported quantity decreases, pointing towards zero at a value $\mu_{c}^{1}$. This is unambiguously a symmetric phase (remember the discussion at the end of the previous section) and therefore we can interpret the plotted points in this region as the inverse diquark susceptibility.

From $\mu_{c}^{1}$ to $\mu_{c}^{2}$ this quantity stays (almost) zero and then it starts to grow. Following again the discussion at the end of the previous paragraph we have checked that, inside the statistical errors, the numerical data in this region are consistent with a non-vanishing diquark condensate.

We interpret these results as signals of two phase transitions. In table II we report the values of the two critical chemical potential (from the larger volume data set) and half the pion mass.

\begin{tabular}{|c|c|c|c|}
\hline$m$ & $\mu_{c}^{1}$ & $\mu_{c}^{2}$ & $\frac{m_{\pi}}{2}$ \\
\hline 0.025 & $0.20(3)$ & $0.8(1)$ & 0.19 \\
0.05 & $0.25(3)$ & $0.8(1)$ & 0.27 \\
0.2 & $0.46(1)$ & $0.9(1)$ & 0.48 \\
\hline
\end{tabular}

Table II

The critical value $\mu_{c}^{1}$ depends on the quark mass and is coincident with the position of the peak in the chiral susceptibility, while $\mu_{c}^{2}$ is essentially independent of the quark mass. This second phase transition may be identified with the saturation phase transition that occours in any theory at finite density on a discrete lattice. In fact (referring, for example, to the results for the number density in [14]) at $\mu=0.8$ we have that the lattice system is almost filled with baryons. Assuming that the phase structure found at $\beta=0$ remains qualitatively unchanged at larger values of $\beta$, this picture does not support the existence of a third physical phase as claimed by the authors of [5].

Considering both the chiral and diquark results one may deduce the following phase structure: increasing the density (i.e. the chemical potential) the system moves through two phases: the first one in which the chiral condensate is nonzero and the diquark condensate is zero, similar to the standard zero density phase, and, at large enough density, a phase in which the chiral 
condensate becomes approximatively zero (exactly zero only in the $m \rightarrow 0$ limit) and the diquark condensate becomes nonzero. Analysing this phase space on the basis of the symmetries of the theory one may conclude that increasing the chemical potential there is a phase transition at which the chiral symmetry is restored and the baryon number symmetry is broken. The value of the chemical potential at which this transition occours coincides with half the pion mass.

What emerges here is a good quantitative agreement with available data for finite density $S U(2)$. Not only the phase structure that emerges from our simulations is well compatible with the one obtained in [4, 5] but also the critical points are in good agreement for several values of the quark mass.

To have a better understanding of the nature of the diquark condensation transition and also a more precise determination of the critical chemical potential we have analysed the critical behaviour of the susceptibility in the symmetric phase by fitting the data at $m=0.2$ with a standard form for a second order phase transition:

$$
\chi^{-1}=C\left(\mu_{c}-\mu\right)^{\gamma_{d}}
$$

where $C$ is an arbitrary constant and $\gamma_{d}$ the critical exponent. In figure 3 we plot our data for the two lattice volumes $4^{4}$ and $6^{4}$ and superimpose the fit of the larger lattice data, with parameters $\gamma_{d}=0.63(3), \mu_{c}^{1}=0.46(1)$ (leftmost part of the figure).

We can see that, apart from a region near the phase transition in the small lattice, relation (4) fits very well our data, and the value of the critical chemical potential agrees very well with half the pion mass. Also the behaviour near the saturation transition can be described by a similar formula. We report in the figure also the result of this fit.

Before concluding our analysis we compare the results of our simulations with the more recent ones reported in [2], where a detailed analysis of the low energy Lagrangian for QCD-like theories with fermions in the pseudoreal representation has been performed. In Figure 4 we report, our results (symbols) appropriately rescaled to allow a direct comparison with the analytical predictions (lines) [2]; an almost perfect agreement between numerical data and low energy Lagrangian prediction does exist up to $\mu / m_{\pi} \simeq 0.6$. The subsequent deviation can be ascribed to the appearance of saturation effects in the numerical results. We present only data for $m=0.2$ but similar conclusions can be depicted also at different (smaller) quark masses.

In conclusion we have found evidence for the existence of a phase transition which separates a low density phase, with baryon number conservation 
and spontaneous chiral symmetry breaking, from a high density one, where chiral symmetry is restored, baryon number is spontaneously broken and a non vanishing diquark condensate appears. Furthermore the numerical results are compatible with a continuous phase transition. This evidence corroborates the expectations for a phase with diquark condensation. Notwithstanding that our calculation at strong coupling is far from the continuum limit, the very good agreement between our results and the predictions of the continuum low energy effective lagrangian strongly suggests a very weak $\beta$ dependence.

\section{Acknowledgements}

R.A. thanks S. Hands for useful discussions. This work has been partially supported by CICYT (Proyecto AEN97-1680) and by a INFN-CICYT collaboration. The Consorzio Ricerca Gran Sasso has provided part of the computer resources needed for this work.

\section{References}

[1] M. Alford, K. Rajagopal, F. Wilczek, Phys. Lett. B422 (1998) 247; Nucl. Phys. B537 (1999) 443; R. Rapp, T. Schaefer, E.V. Shuryak, M. Velkovsky, Phys. Rev. Lett. 81 (1998) 53; T. Schafer, F. Wilczek, Phys. Rev. Lett. 82 (1999) 3956.

[2] J.B. Kogut, M.A. Stephanov, D. Toublan, J.J.M. Verbaarschot, A. Zhitnitsky, hep-ph/0001171.

[3] S. Hands, J.B. Kogut, M.P. Lombardo, S.E. Morrison, Nucl. Phys. B558 (1999) 327.

[4] E. Dagotto, A. Moreo, U. Wolff, Phys. Rev. Lett. 57 (1986) 1292.

[5] E. Dagotto, A. Moreo, U. Wolff, Phys. Lett. B186 (1987) 395.

[6] J.U. Klaetke, K.H. Mütter, Nucl. Phys. B342 (1990) 764.

[7] S. Hands, S. Morrison, proceedings of Understanding Deconfinement in QCD, Trento, March 1999, hep-lat/9905021.

[8] S. Morrison, S. Hands, proceedings of Strong and Electroweak Matter 98, Coepnhagen, Dec. 1998, hep-lat/9902012. 
[9] V. Azcoiti, G. Di Carlo, A. Galante, A.F. Grillo, V. Laliena, C. E. Piedrafita, Phys. Lett. B353 (1995) 279.

[10] V. Azcoiti, V. Laliena, X. Luo, Phys. Lett. B354 (1995) 111.

[11] R. Aloisio, V. Azcoiti, G. Di Carlo, A. Galante, A.F. Grillo, to be published in the proceedings of the "International Workshop on NonPerturbative Methods and Lattice QCD", Guangzhou, China, 15-21 May 2000, hep-lat/0007018.

[12] V. Azcoiti, G. Di Carlo, A.F. Grillo, Phys. Rev. Lett. 65 (1990) 2239; V. Azcoiti, A. Cruz, G. Di Carlo, A.F. Grillo and A. Vladikas, Phys. Rev. D43 (1991) 3487; V. Azcoiti, G. Di Carlo, L.A. Fernandez, A. Galante, A.F. Grillo, V. Laliena, X.Q. Luo, C.E. Piedrafita and A. Vladikas, Phys. Rev. D48 (1993) 402; V. Azcoiti, G. Di Carlo, A. Galante, A.F. Grillo, V. Laliena, Phys. Rev. D50 (1994) 6994.

[13] H. Kluberg-Stern, A. Morel, B. Petersson, Nucl. Phys. B215 (1983) 527.

[14] R. Aloisio, V. Azcoiti, G. Di Carlo, A. Galante, A.F. Grillo, Nucl. Phys. B564 (2000) 489. 
- Figure 1: Chiral suceptibility at $m=0.025$ and $m=0.05$ in $4^{4}, 6^{4}$ lattices.

- Figure 2: Inverse diquark susceptibility at $m=0.05$ in $4^{4}, 6^{4}$ lattices.

- Figure 3: Inverse diquark susceptibility at $m=0.2$ and fit of $6^{4}$ data using formula (4).

- Figure 4: Low energy Lagrangian predictions (lines) and our data (symbols) for chiral condensate, diquark condensate and number density at $m=0.2$ and $j=0.1 m$ vs. $\mu / m_{\pi}$. $6^{4}$ data are squares and circles, $4^{4}$ data are diamonds. 
स्र

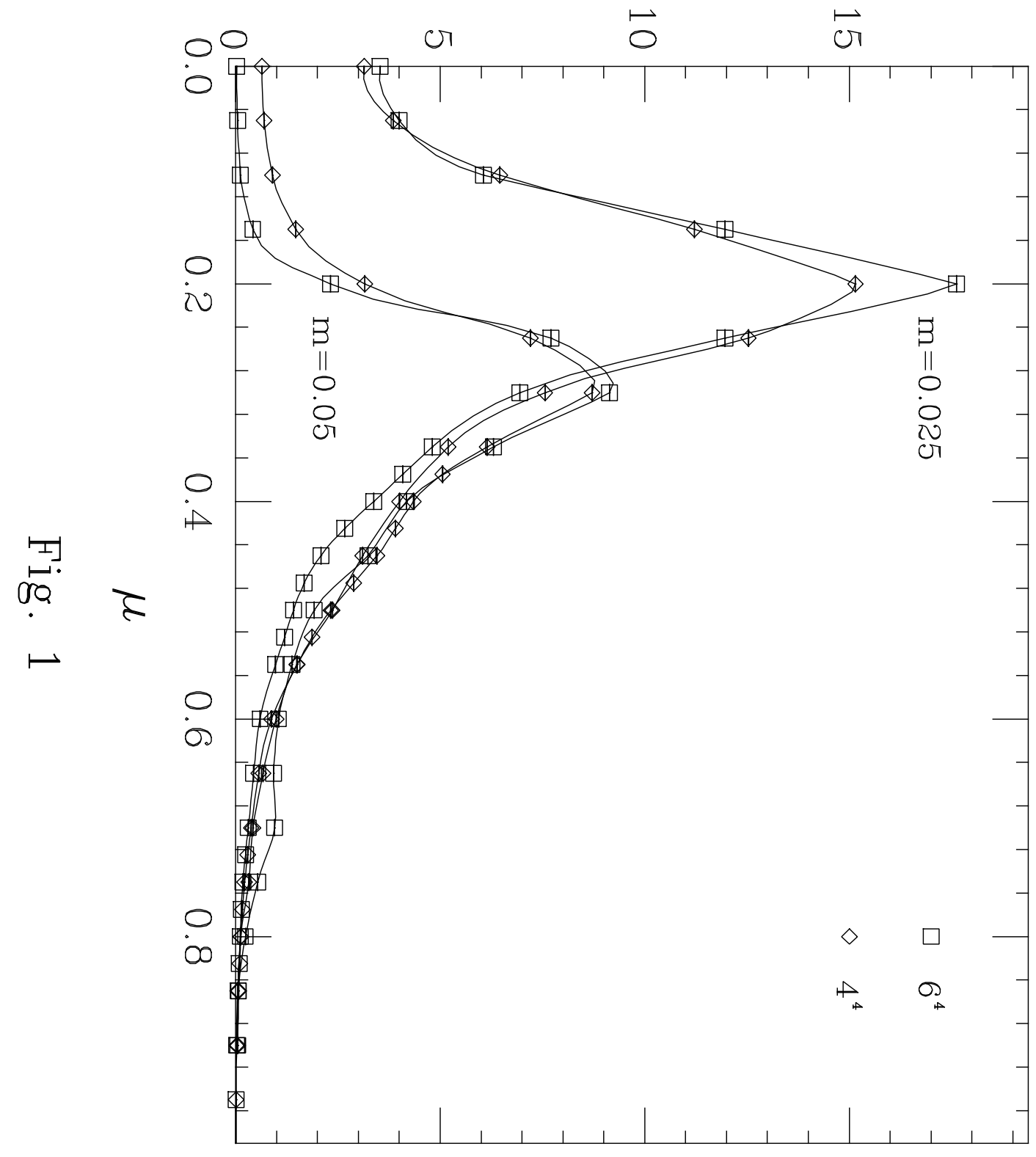




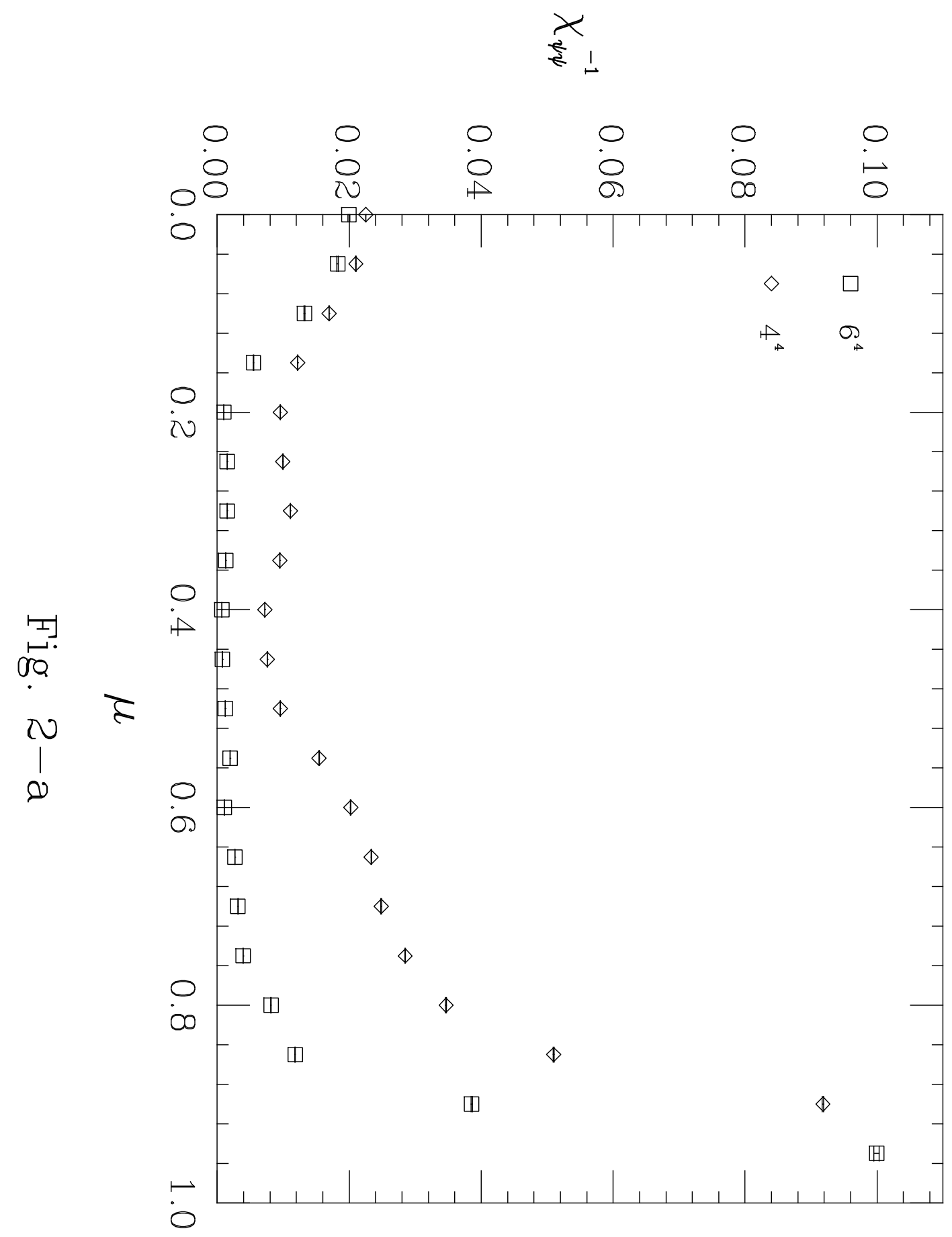




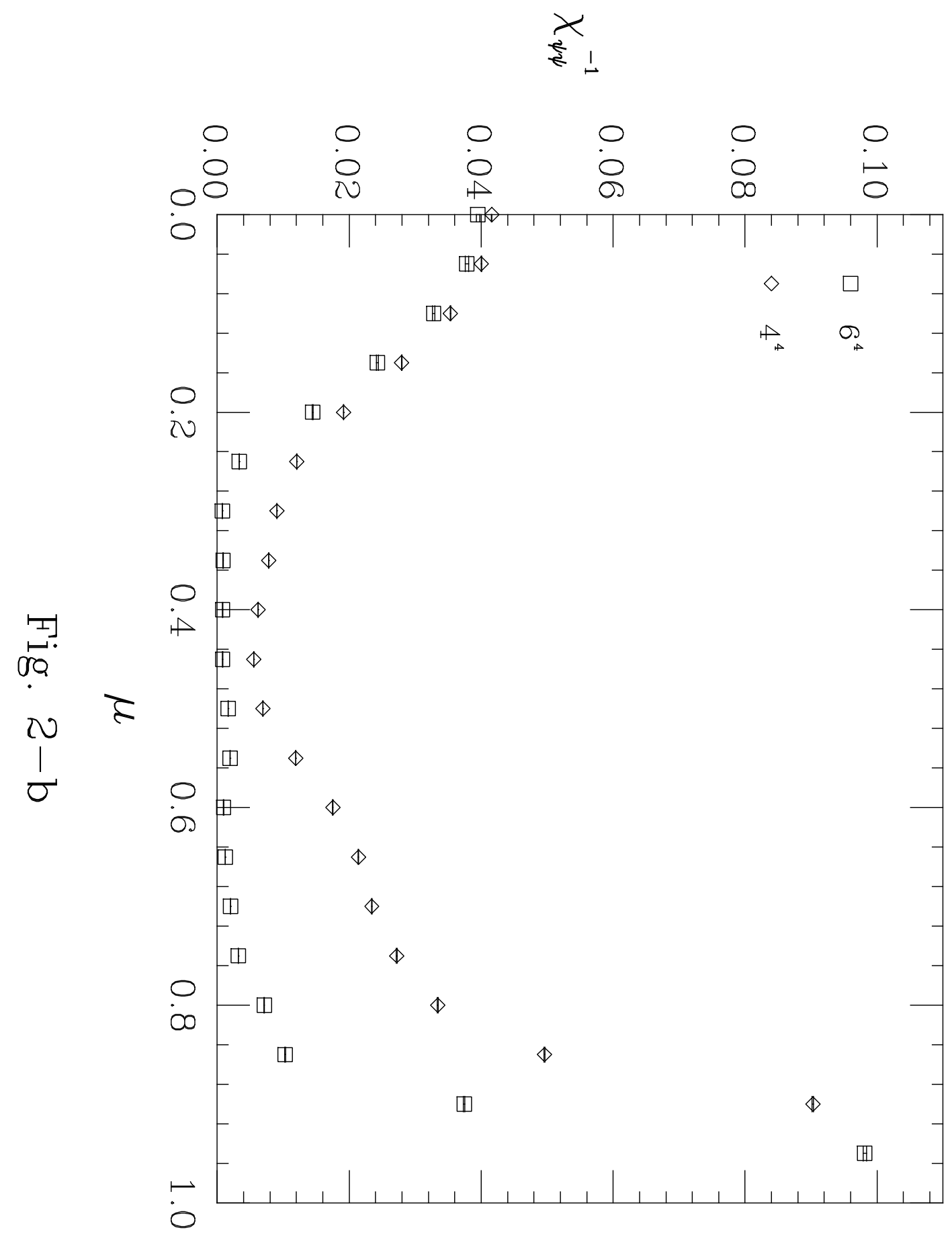




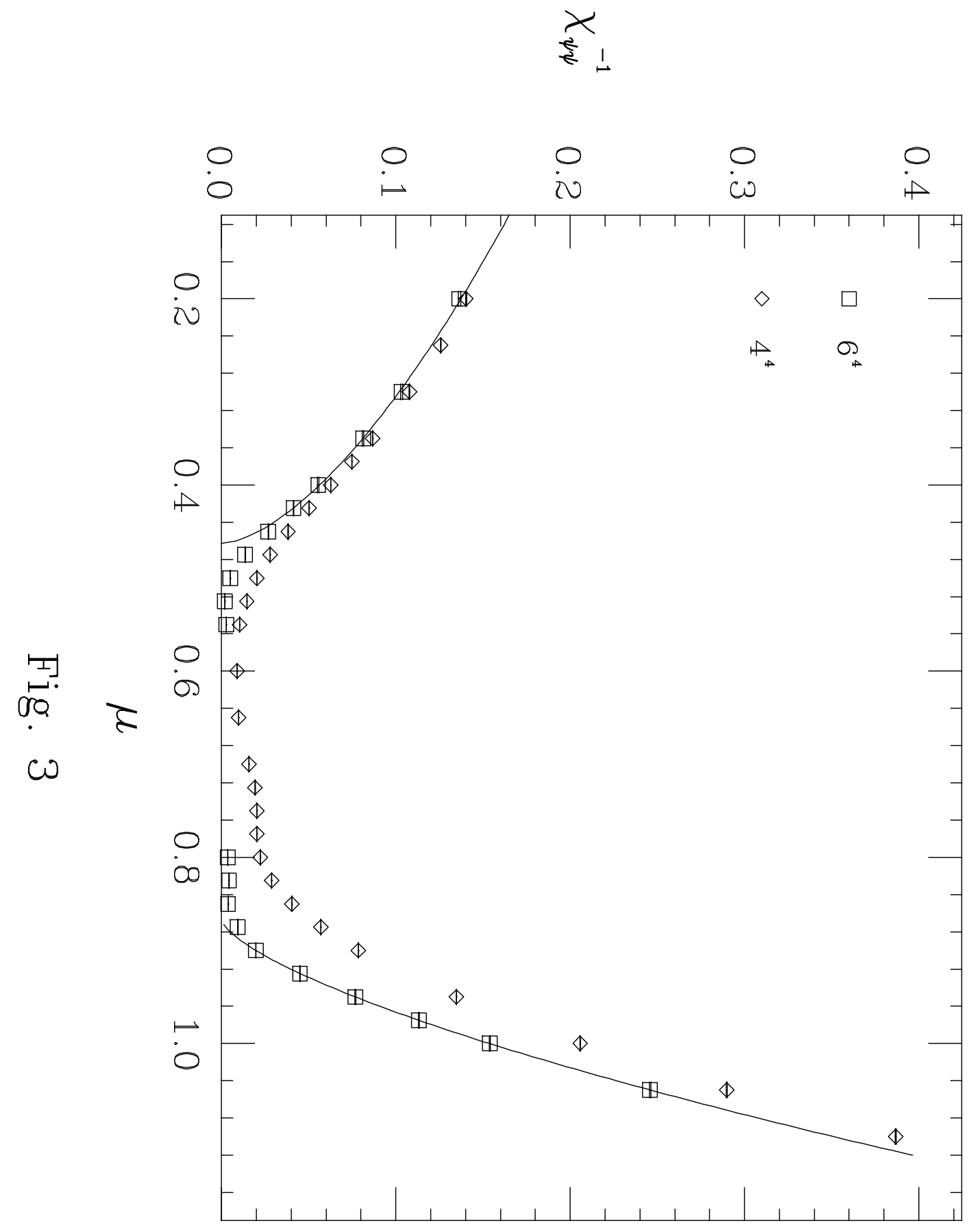




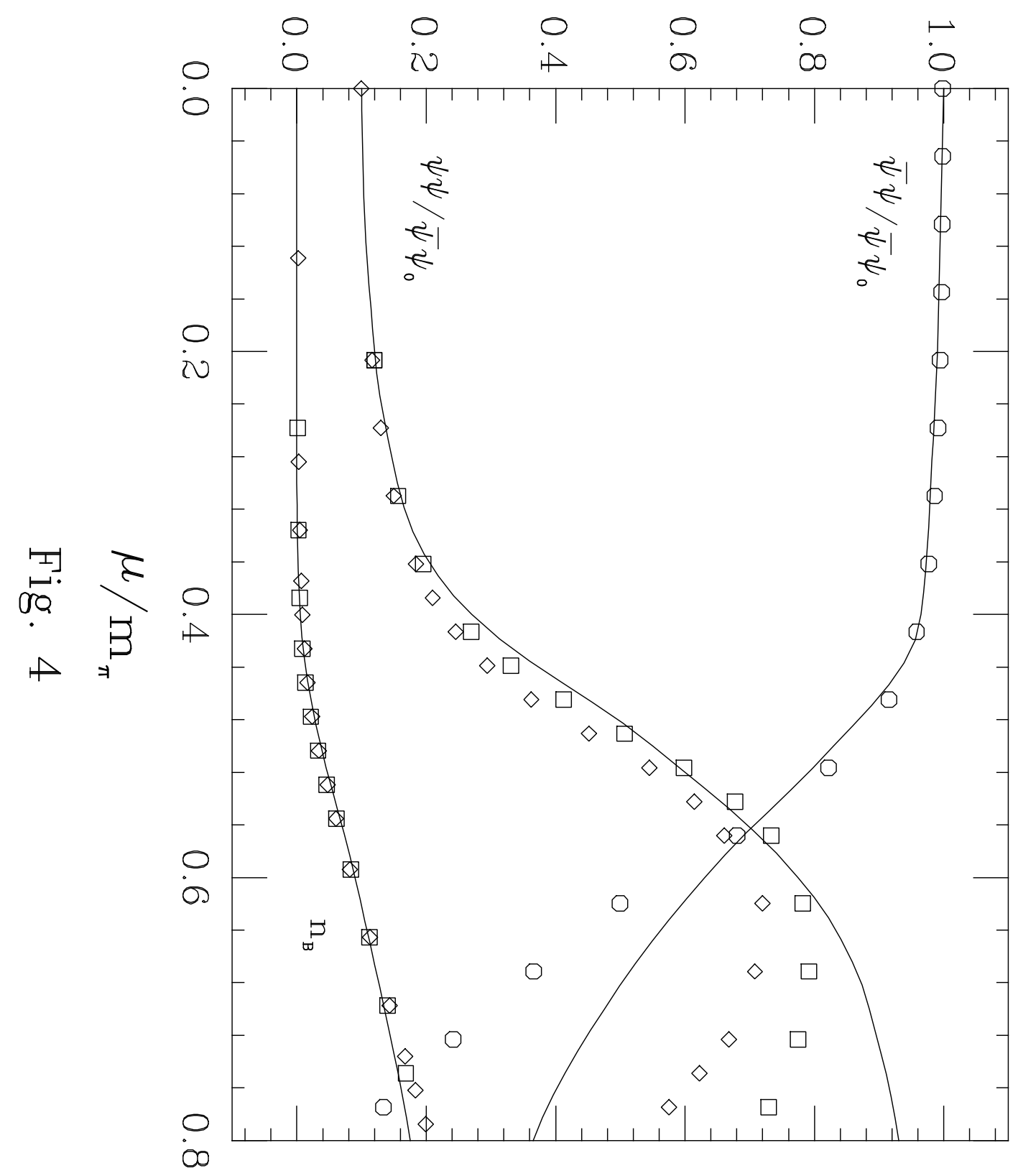

